ISSN 1112-9867

Available online at $\quad$ http://www.jfas.info

\title{
CREATION OF A SYSTEM OF SIGNS TO IDENTIFY THE USER ON THE DYNAMICS OF REPRODUCTION SIGNATURES
}

\author{
D. V. Lapin*, T. I. Lapina \\ Southwest State University 305040, Kursk, st.50 years of October, 94
}

Published online: 08 August 2017

\begin{abstract}
Describes a method of creating a system of signs to identify the user for authentication on the dynamics of ink handwriting using a multi-touch sensor. The result is a reduction of the computational complexity of classification when creating access control systems.
\end{abstract}

Keywords: analysis and classification of signals, user identification, access control systems.

\section{INTRODUCTION}

The task of creation of the centralized management information systems, the integrated security arrangements of objects, systems access control to information resources, development of network communications requires continuous interaction with the equipment, services, services and the software and in all these cases the warranty of confirmation of the identity of the subject entering information exchange is required. Such warranty is provided in the course of user registration in this or that system and performing procedures of identification, authentication and authorization.

Need of development of a complex of technical means and procedures of reliable identification, authentication and authorization of users for information networks of data transfer is a consequence of such tendencies [6].

The methods applied to determination of authenticity of the personality use different technologies of protection by which development the main directions are use of various sensors for receiving basic data for identification, creation of architecture of monitoring devices and connection them to the computer.

Author Correspondence, e-mail: author@gmail.com

doi: http://dx.doi.org/10.4314/jfas.v9i2s.83 
Accuracy of identification in such systems is largely determined by way of receiving basic data as which the priority is given to biometric measurements, such as hand-written handwriting, voice, drawing of blood vessels on an arm and so forth and also the used methods of statistical data processing, the factorial analysis and recognition of images.

The feature of use of hand-written handwriting for identification in comparison with the alternative methods based on the analysis of images consists in need of reproduction of strictly certain sequence of actions, unique for each person. Process of reproduction of the signature is considered in the speaker that provides impossibility of substitution of the entered sample. Besides the procedure of identification for dynamics of handwriting allows to register keywords, different on the writing, for each subscriber of system. This property provides additional protection against an information compromise in case of identification.

In this article justification and realization of approaches to formation of system of signs for carrying out identification of the personality on dynamics of reproduction of the signature is considered [2].

For obtaining the measured biometric data the specialized device which is the multicomponent sensor of movements [3] allowing for $n$ directions of sensor movement in the plane to record measurements of static or smoothly changing pressure on the writing unit (Fig. 1).

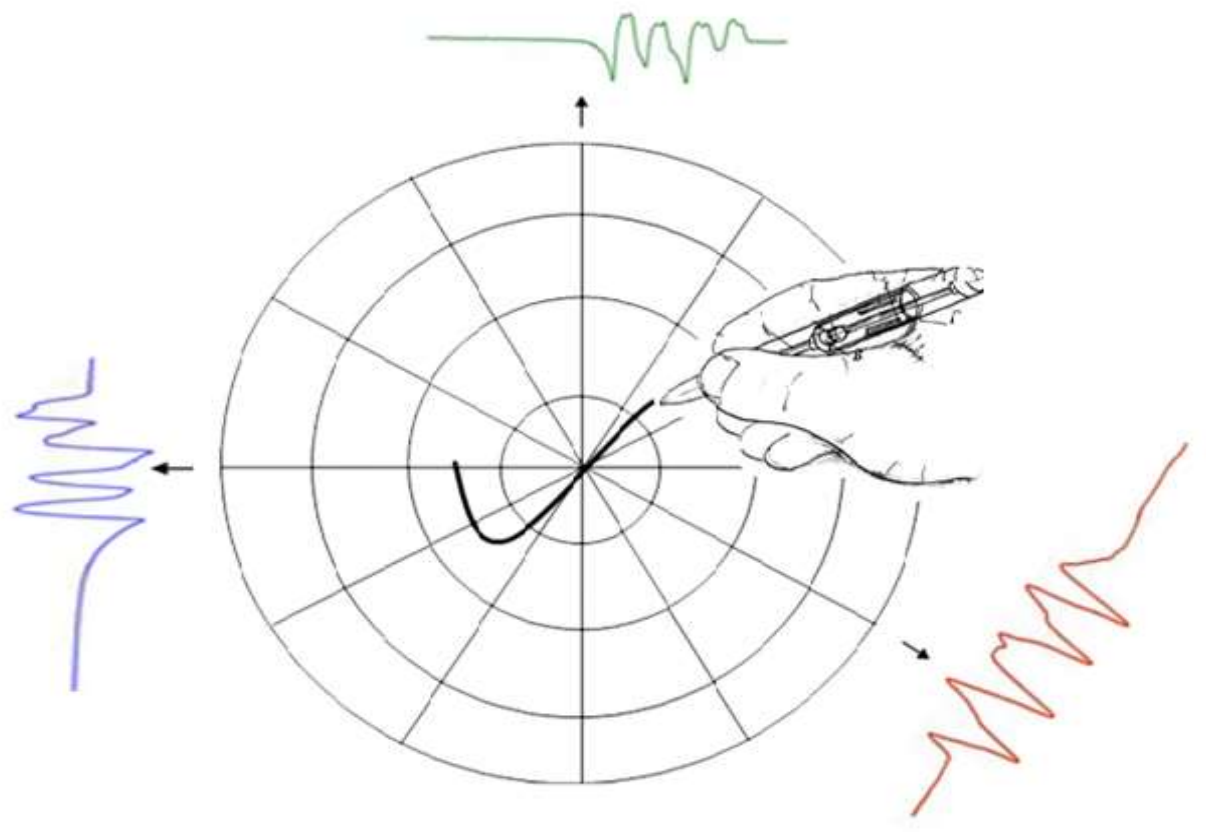

Fig.1. Multicomponent sensor of movements 
The obtained biometric data represent measurements of pressure upon the writing knot when moving on $n$ to the directions of the sensor of movements when writing a password phrase, that is curve changes of pressing the writing knot on axes of coordinates of $X 1(t) . X n(t)$. The device of measurement of parameters of pressure when moving makes fixing of signals with the subsequent digitization of measurements [4] is given in fig. 2.

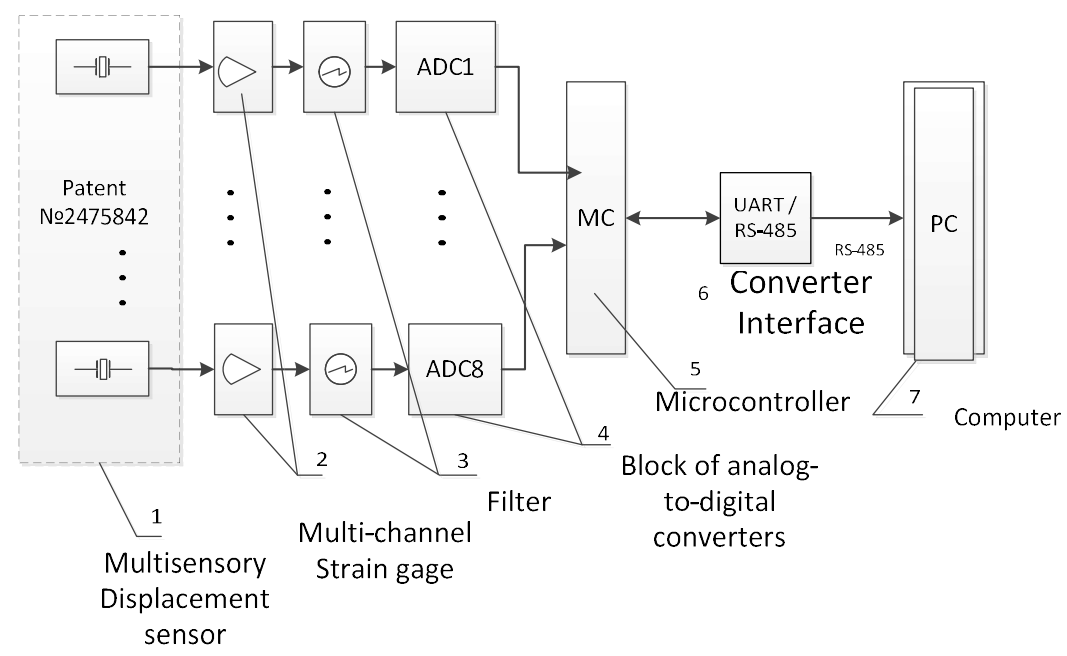

Fig.2. Devices for measurement of parameters of pressure when moving in $N$ directions of the multitouch sensor of movements

At the same time specific features of reproduction of the signature by the user are reflected in frequency structure of functions $\mathrm{x}_{1}(\mathrm{t}), \mathrm{x}_{2}(\mathrm{t}), \ldots, \mathrm{x}_{\mathrm{n}}(\mathrm{t})$, and the task of the biometric analysis comes down to extraction of this information [5], (fig. 3). 


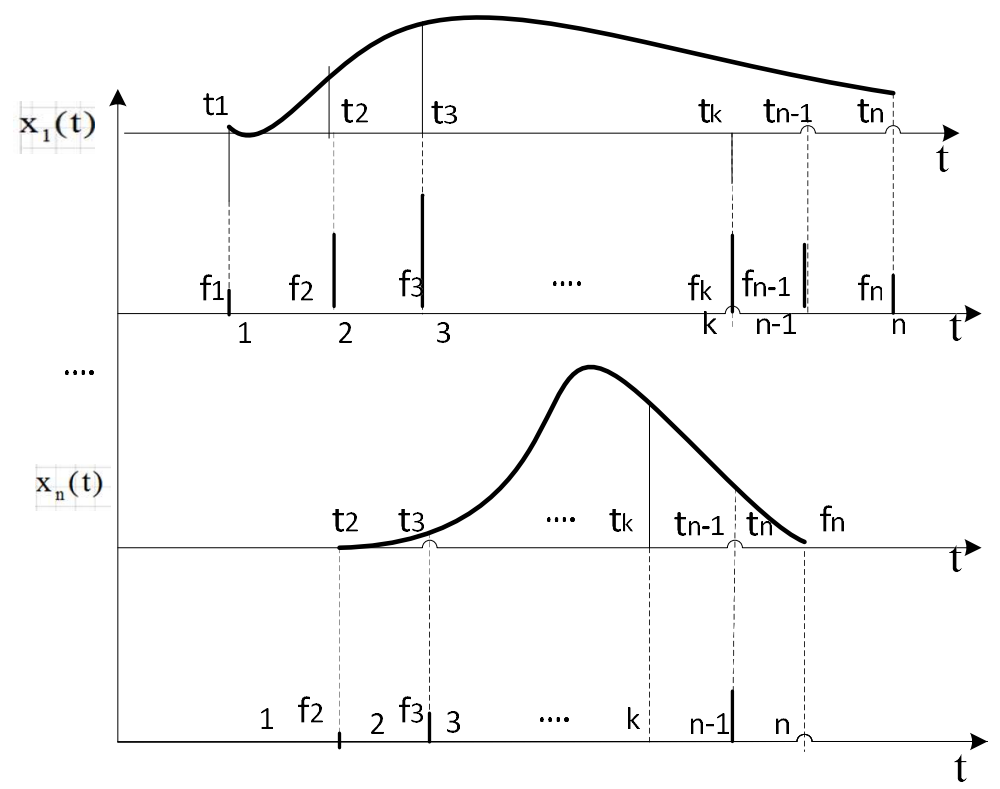

Fig.3. Signals of pressure upon the writing knot when moving the sensor

Use of representation of basic data in the form of a multicomponent discrete signal allows to make the analysis of each fragment of decomposition of a signal as separate that simplifies a problem of a marking and the analysis of an initial image of a signal.

Realization of functions $\mathrm{x}_{1}(\mathrm{t}), \mathrm{x}_{2}(\mathrm{t}), \ldots, \mathrm{x}_{\mathrm{n}}(\mathrm{t})$ can be treated as the casual processes proceeding in the range of $\mathrm{T}$ time, appropriate time of reproduction of the signature.

Formations of a biometric image it is, as a rule, carried out with use of linear functionalities, such as Fourier, Walsh, Haar's orthogonal functionalities.

The system of orthogonal functions of Haar can be used as basic at decomposition in evenly meeting Haar's number of the continuous signal of final duration set on a piece $[0, T)$. Application of functions of Haar is most effective for the analysis of signals with strongly expressed local features in the form of short-term splashes and fluctuations. This results from the fact that approximation of these splashes and fluctuations is carried out by limited number of the components of a row located in the corresponding part of an interval [Oh, T).

Haar's functions har $(r, m, t)$ it is possible to receive from a recurrence relation:

har $(0,0, t)=1, t \in[0,1)$; 


$$
\operatorname{har}(r, m, t)=\left\{\begin{array}{c}
2^{r / 2}, \text { if } \begin{array}{c}
m-1 \leq t<m-1 / 2 \\
2^{r}
\end{array} \\
-2^{r / 2}, \text { if } m-1 / 2 \leq t<\begin{array}{l}
m \\
0,
\end{array} ; \\
2^{r} \quad \text { At the other } \quad t \in[0,1),
\end{array}\right.
$$

where $0 \leq r<\log _{2} N_{\text {и }} 1 \leq m \leq 2^{r}$.

The discretization of the Haar function system leads to the Haar matrix $\mathrm{H}^{*}(\mathrm{n})$, where $\mathrm{n}$ $=\log _{2} N$. Thus, for $N=8$, the Haar matrix $\mathrm{H}^{*}$ (3) will have the form

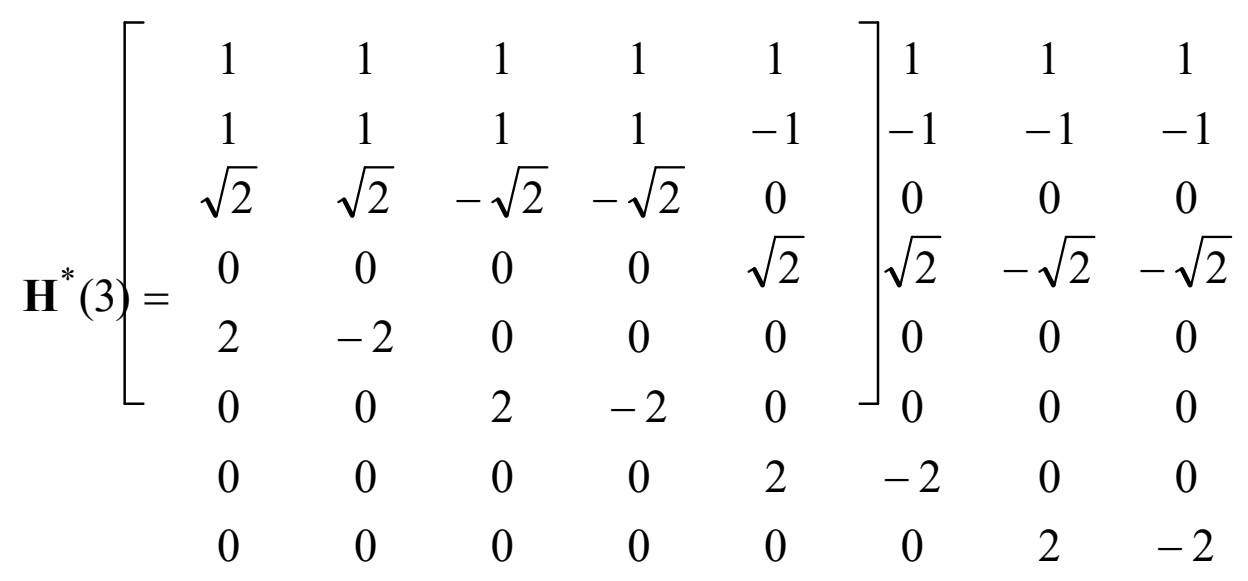

Every line of a matrix (2) is discrete function of $\operatorname{Haar} \operatorname{Har}(r, m, t)$.

For example, in case of two-dimensional discrete transformation of Haar it is required to execute transformation of a matrix $\mathrm{N} \times \mathrm{N}$ of discrete values of measurements of the multicomponent sensor of movements. Operations at first are carried out every line of a matrix, and then the same operation is carried out with each column of result (3):

$$
\mathrm{PW}=\left(\begin{array}{cccc}
18 & 14 & 12 & 4 \\
10 & 6 & 8 & 8 \\
16 & 4 & 8 & 0 \\
12 & 0 & 4 & 4
\end{array}\right) \times \frac{1}{4} \times\left(\begin{array}{cccc}
1 & 1 & 2 & 0 \\
1 & 1 & -2 & 0 \\
1 & -1 & 0 & 2 \\
1 & -1 & 0 & -2
\end{array}\right)=\left(\begin{array}{cccc}
12 & 4 & 2 & 4 \\
8 & 0 & 2 & 0 \\
7 & 3 & 6 & 4 \\
5 & 1 & 6 & 0
\end{array}\right)
$$

where, a matrix $\mathrm{P}$ - matrixes of $\mathrm{N} \times \mathrm{N}$ of values of discrete measurements; $\mathrm{W}$ - orthonormal function of Haar of the fourth order[5].

Then the matrix of transformation of columns is transposed, then multiplied by a reformative matrix then the result is again transposed (4):

$$
T=\left((P W)^{T} \times W\right)^{T}=W^{T} \times P \times W,
$$


the sign ()$^{T}$ means transposing.

Thus, two-dimensional discrete transformation of an initial matrix of $\mathrm{P}$ will be (5):

$$
T=W^{T} P W=\frac{1}{4} \times\left(\begin{array}{cccc}
1 & 1 & 1 & 1 \\
1 & 1 & -1 & -1 \\
2 & -2 & 0 & 0 \\
0 & 0 & 2 & -2
\end{array}\right) \times\left(\begin{array}{cccc}
12 & 4 & 2 & 4 \\
8 & 0 & 2 & 0 \\
7 & 3 & 6 & 4 \\
5 & 1 & 6 & 0
\end{array}\right)=\left(\begin{array}{cccc}
8 & 2 & 4 & 2 \\
2 & 0 & -2 & 0 \\
2 & 2 & 0 & 2 \\
1 & 1 & 0 & 2
\end{array}\right)
$$

The transformed matrix of $\mathrm{T}$ contains average value of all elements of an initial matrix in the left top corner, and other elements correspond to differences. The elements which are spaced far apart from the left top corner correspond to the level of more exact specification, that is more high-frequency elementary waves.

The received matrix $\mathrm{T}$ coefficients of two-dimensional discrete transform of Haar is used further as an identification image for the subsequent procedure of authentication to which the grade level of system precedes [2].

We will interpret the received matrix as a multidimensional time row. Then the problem of identification comes down to estimation of statistical characteristics of temporary ranks and comparison with a standard. In this case the problem of distinction of signals can be reduced to a problem of change of structure of casual process or a temporary row.

The scheme of the computerized system of biometric identification is provided on fig. 4 . 


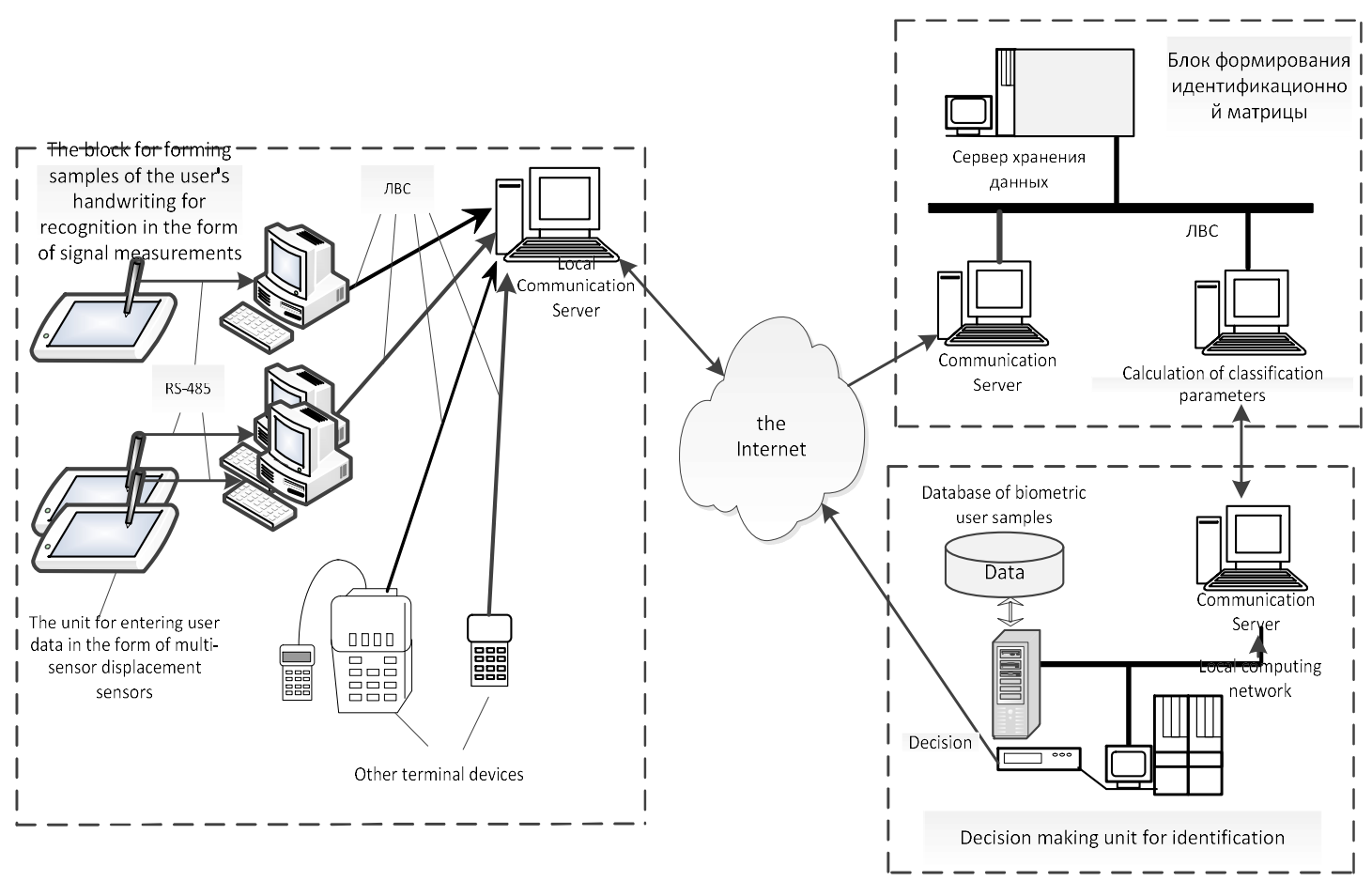

Fig.4. The scheme of the computerized system of biometric identification

The created biometric image is used in case of implementation of procedures of authentication of the registered user. One of simple decisive rules is use of a measure of Hamming for bit representation of a vector of $\mathrm{V}=\left(\mathrm{v}_{1}, \mathrm{v}_{2} \ldots, \mathrm{v}_{\mathrm{k}}\right)$.

Let the system in case of identification realize measurement of a vector of $V=\left(v_{1}, v_{2} \ldots, v_{k}\right)$ consisting of $k$ of biometric parameters. Let at a stage of registration (training) the authorized user showed $N$ signatures and, respectively, we have $N$ implementations of vectors of $\mathrm{V}_{\mathrm{i}}$.

Having analysed the available realization of vectors of biometric parameters, it is possible to find a characteristic interval of change of each concrete parameter $\left[\min \left(\mathrm{v}_{\mathrm{j}}\right), \max \left(\mathrm{v}_{\mathrm{j}}\right)\right]$. If now at hit of the $\mathrm{v}_{\mathrm{j}}$ parameter in an interval $\left[\min \left(\mathrm{v}_{\mathrm{j}}\right), \max \left(\mathrm{v}_{\mathrm{j}}\right)\right]$ to appropriate $\mathrm{e}_{\mathrm{j}}=0$, and at loss of $\mathrm{v}_{\mathrm{j}}$ from an interval $\left[\min \left(\mathrm{v}_{\mathrm{j}}\right), \max \left(\mathrm{v}_{\mathrm{j}}\right)\right]$ to appropriate $\mathrm{e}_{\mathrm{j}}=1$, then we will receive Hamming's vector. For the registered user this vector has to consist practically of one zero. For the unregistered user showing other biometric parameters, Hamming's vector will have a large number of discrepancies - units.

Then absolute value of distance of Hamming $-E_{u}$ to a biometric standard should be defined as the total number of losses of measurements for intervals of admissible values of a 
biometric standard. Hamming's distance $-\mathrm{E}_{\mathrm{u}}$ always positively can also change from 0 to $k$ (where $k$ is a number of controlled biometric parameters).

At the capacity of training selection of 5 and more examples become expedient calculation of population mean of values of parameters $m\left(v_{i}\right)$ of their dispersions $\sigma^{2}\left(v_{i}\right)$. In this case the value of the minimum and maximum borders can be calculated as follows (6):

$$
\begin{aligned}
& \min \left(v_{\mathrm{i}}\right)=\mathrm{m}\left(v_{\mathrm{i}}\right)-\mathrm{S}\left[\mathrm{L},\left(1-\mathrm{P}_{1}\right)\right] \cdot \sigma^{2}\left(v_{\mathrm{i}}\right), \\
& \min \left(v_{\mathrm{j}}\right)=\mathrm{m}\left(v_{\mathrm{j}}\right)+\mathrm{S}\left[\mathrm{L},\left(1-\mathrm{P}_{1}\right)\right] \cdot \sigma^{2}\left(v_{\mathrm{j}}\right)
\end{aligned}
$$

where L - number of the examples used when training: $\mathrm{P}_{1}$, - a preset value of probability of errors of the first sort; $\mathrm{S}\left(\mathrm{L} .\left(1-\mathrm{P}_{1}\right)\right)$ - Styyudent's coefficient.

When using rather large number of controlled biometric measurements distribution of values of a measure of Hamming is close to normal. In this case the threshold value of a measure of Hamming $\mathrm{E}_{\mathrm{k}}$ can be determined through population mean and dispersion of values of a measure of Hamming for "its" user by the $\mathrm{E}_{\mathrm{c}}(6)$ :

$$
\mathrm{E}_{\mathrm{k}}=\mathrm{m}\left(\mathrm{E}_{\mathrm{c}}\right)+\mathrm{S}\left[\mathrm{L},\left(1-\mathrm{P}_{1}\right)\right] \cdot \sigma^{2}\left(\mathrm{E}_{\mathrm{c}}\right)
$$

where $\mathrm{S}\left[\mathrm{L},\left(1-\mathrm{P}_{1}\right)\right.$ - Styyudent's coefficient set from among the used examples of $L$ and the size of an error of the first sort (probabilities of $\mathrm{P}_{1}$ of false refusal to "its" user [5].

\section{CONCLUSIONS}

1. Receiving measurements of biometric data in the form of a multicomponent signal allows to make the analysis of each fragment of decomposition of a signal as separate that simplifies a problem of a marking and the analysis of an initial image of a signal.

2. The technique of formation of a biometric image on the basis of allocation of dynamic characteristics of signals of the multitouch sensor of the movements transformed by the device of measurement of parameters of the writing knot to a matrix of discrete counting with use of transformation of Haar is offered. Application of functions of Haar allows to consider local features of signals in the form of short-term splashes and doesn't demand considerable computing expenses that allows to provide formation of high-informative dynamic signs. 


\section{REFERENCES}

1. Lapin, D.V. The method of biometric user authentication in computerized access control systems / D.V. Lapin, T.I. Lapina, N.N.Epishev // Proceedings of SPIIRAS.- SPb .: SPIIRAN, 2013 .- Issue No. 4 (27), p.189-199

2. Lapin, D.V. Approach to classification of digital signals in access control systems / D.V. Lapin E.A., Petrik, T.I. Lapina//the Information and measuring and controlling systems. 2013. t.11.№9. page 58-64.

3. Patent No. 2475842 of the Russian Federation Digital multicomponent sensor of relocation / Milyh V. A., Lapin D.V., Lapina T.I., Request No. 2011142722/08 of 21.10.2011, опубл. 20.02.2013, Bulletin No. 5.

4. Patent No. 2475699 of the Russian Federation Device of measurement of parameters of a writing node / Milyh V.A., Lapin D.V., Lapina T.I. Request No. 2011113800/28, 08.04.2011, опубл. 20.02.04.2013, Bulletin No. 5.

5. The patent No. 469397 of the Russian Federation the Method of biometric authentication on handwriting in the computerized control system of accesses / Milyh V. A., Lapin D.V., Lapina T.I., the Request No. 2011140031/08, 30.09.2011, опубл. 10.12.2012, Bulletin No. 34.

6. Ivanov A.I. Biometric identification of the personality on dynamics of subconscious movements / A.I. Ivanov//the Monograph. - Penza: Penz publishing house. state. un-that, 2000. $\square 188$ pages.

\section{How to cite this article:}

Lapin D V, Lapina T I. Creation of a system of signs to identify the user on the dynamics of reproduction signatures. J. Fundam. Appl. Sci., 2017, 9(2S), 1090-1098. 